\title{
COMPARISON BETWEEN 40kb/s ADPCM ALGORITHMS
}

\author{
MUHANNED AL-RAWI ${ }^{1}$ (Corresponding Author), MUAAYED AL-RAWI ${ }^{2}$ \\ ${ }^{1}$ University of Ibb, Yemen, ${ }^{2}$ AL-Mustansiryia University, Iraq \\ E-mail: muhrawi@yahoo.com,muaayed@yahoo.com
}

\begin{abstract}
This article deals with developing new modified algorithms of 40kb/s ADPCM. Beside the standard algorithm, three algorithms are developed to reduce the ADPCM distortion. The performances of all algorithms are evaluated with QAM signal at speed of $9.6 \mathrm{~kb} / \mathrm{s}$. The outcomes demonstrate that the first proposed algorithm outperforms the other algorithms.
\end{abstract}

Keywords: ADPCM, QAM modem

\section{INTRODUCTION}

Due to the importance of representing speech, and data signals in digital form, different techniques of signal coding and in particular speech coding were developed. Adaptive Differential Pulse Code Modulation (ADPCM) [1] is one of those techniques which attracted attention due to its unique merits. These merits relate to performance, economy, and applications flexibility.

ADPCM has wide applications in telecommunication networks. The recent applications of ADPCM were published as U.S. patents [2-5]. In any application, voiceband information signals and speech signals must be sent, and sufficient transmission execution must be kept for speech as well as for rapid voiceband information signals. In any case, speech and voiceband information signals have diverse qualities in time area and frequency area. The contrasts between them increment the trouble of satisfactory transmission execution for both.

The time area qualities of the two signals can represented by looking at their autocorrelation capacities. The autocorrelation elements of speech and voiceband information signals have estimated estimations of 0.85 and 0.2 separately for the waveform sampling at $8000 \mathrm{sample} / \mathrm{sec}$, which is the sampling rate of standard ADPCM. Then again, the frequency area attributes of speech and voiceband information signals can be delineated by looking at their spectra. The frequency range of speech is packed in the range 200$800 \mathrm{~Hz}$, while, by examination, the frequency range of $9.6 \mathrm{~kb} / \mathrm{s}$ modem is level in the scope of $500-3000 \mathrm{~Hz}$.

The major issue of ADPCM is attributed to its serious distortion when used to code vioceband data signal at high speed. Two ways were suggested to overcome this issue. The first way is to modify the ADPCM algorithm
[6-9], while, the second way suggested to modify the data transmission model [10-12].

\section{STRUCTURE OF ADPCM}

\subsection{General structure}

The general structure of ADPCM codec is illustrated in Figure 1. The two main elements form the algorithms: an adaptive quantizer and an adaptive predictor. Figure 1 also shows the connection between encoder and decoder. The difference between them is that the encoder contains adaptive quantizer $(\mathbf{Q})$ and inverse adaptive quantizer $\left(\mathbf{Q}^{-1}\right)$, while, the decoder contains inverse adaptive quantizer only. The decoder is a subset of the encoder and transmits $r(n)$ instead of $c(n)$. Adaptive predictor calculates an input signal estimate $\hat{\mathrm{s}}(\mathrm{n})$, subtracted from the input $s(n)$, to produce the difference $d(n)$. Adaptive quantizer codes $d(n)$ into a codeword $c(n)$ to be transmitted by the sender. ADPCM decoder makes use of $c(n)$ trying to restore original input $s(n)$. In fact, only $\mathrm{r}(\mathrm{n})$ can be restored, associated to $\mathrm{s}(\mathrm{n})$ by,

$$
r(n)=s(n)+e(n)
$$

Where

$$
e(n)=d q(n)-d(n)=r(n)-s(n)
$$

is the error introduced by the quantizer, and $\mathrm{dq}(\mathrm{n})$ is the output of $\mathbf{Q}^{-1}$.

The convention method of evaluating the performance of $\mathrm{ADPCM}$ is via signal-to-error-ratio $\left(\mathrm{S} / \mathrm{N}_{\mathrm{e}}\right)$ given by

$$
S / N_{e}=E\left[s^{2}(n)\right] / E\left[e^{2}(n)\right]=\sigma_{s}^{2} / \sigma_{e}^{2}
$$

Where $\mathrm{E}$ is the expectation, $\sigma_{\mathrm{s}}^{2}$ is the power of the input signal $\mathrm{s}(\mathrm{n}) \& \sigma_{\mathrm{e}}^{2}$ is the power of the error signal $\mathrm{e}(\mathrm{n})$. 


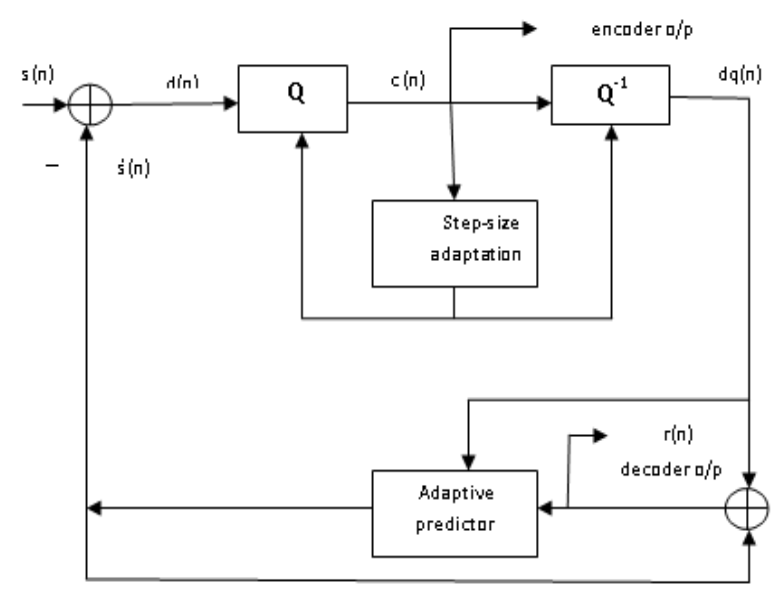

Figure 1. ADPCM Codec

\subsection{Algorithm-1}

This algorithm is the standard one. It uses 5-bit quantizer with sampling rate of 8000 sample/sec to produce coding rate of $5 \times 8000=40 \mathrm{~kb} / \mathrm{s}$. The adaptive predictor consists of two poles and six zeros. The details of the quantizer and predictor can be found in [1].

\subsection{Algorithm-2}

Algorithm-2 is the first new modified proposed algorithm. It uses the same adaptive quantizer as algorithm-1. The difference is identified with the adaptive predictor which comprises of tenth order, zero predictor, fourth order pole predictor, sixteenth order fixed-pole predictor, and offset predictor. The details of this predictor can be found in [6].

\subsection{Algorithm-3}

Algorithm-3 is the second modified algorithm. This algorithm uses 4-bit quantizer with sampling rate of 10000 sample/sec to produce coding rate of $4 \times 10000=40 \mathrm{~kb} / \mathrm{s}$. The details of this quantizer can be found in [1]. The adaptive predictor is as the one of algorithm-1.

\subsection{Algorithm-4}

This is the third modified algorithm which uses the same quantizer as algorithm-3 and the same predictor as algorithm-2.

\section{QAM MODEM DESIGN}

The standard design of Quadrature Amplitude Modulation(QAM) modem has symbol rate of 2400 baud with 4-bit is used to represent each symbol to produce data rate of $2400 \times 4=9.6 \mathrm{~kb} / \mathrm{s}$. The constellation of QAM signal contains $2^{4}=16$-symbol. The design of QAM constellations has strong impact on reducing ADPCM distortion [11]. Four constellations are considered, rectangular, $(5,11),(4,12),(8,8)$ circular as shown in Figure 2.

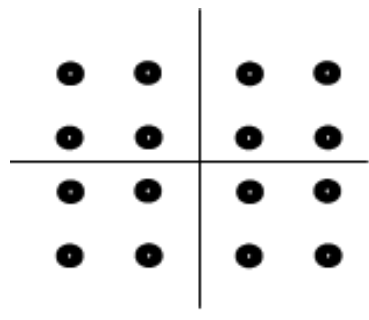

(a) Recturgular

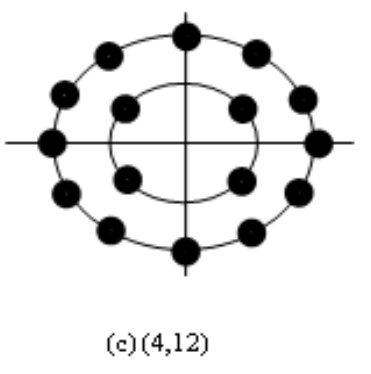

Figure 2. 16-ary QAM constellations

\section{COMMUNICATION SYSTEM MODEL}

An alternative way of evaluating the performance of ADPCM is by developing the communication system shown in Figure 3. The first block in Fig.3 is random generator used to generate binary data (each 4-bit mapped to one symbol of the constellation). The remaining blocks in Fig.3 include QAM modulator, ADPCM codec, Additive White Gaussian Noise (AWGN), QAM demodulator, and simple threshold detector to detect which symbol is transmitted.

The performance of the system is evaluated by sketching symbol error rate (SER) versus signal-to-noise-ratio $(\mathrm{S} / \mathrm{N})$. The SER is given by

$$
S E R=N E D S / N T S
$$

Where NEDS is number of mistaken distinguished symbols and NTS is number of aggregate sent symbols.

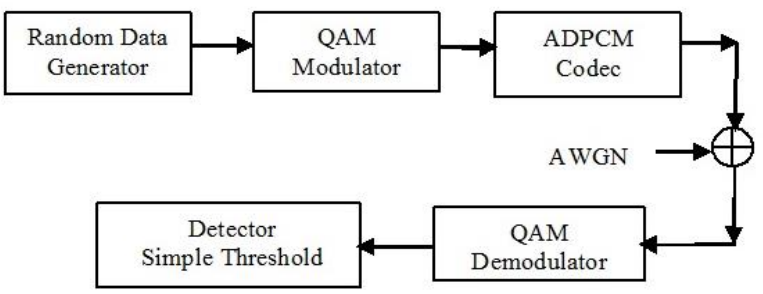

Figure 3. Data transmission

\section{RESULTS}

By using computer simulation, several tests are achieved on the four algorithms of ADPCM using QAM signal to calculate $\mathrm{S} / \mathrm{N}_{\mathrm{e}}$. 
Table 1 presents the values of $\mathrm{S} / \mathrm{N}_{\mathrm{e}}$. It is obvious that algorithm-2 gives better results than algorithm-1 by $2.3 \mathrm{~dB}$ for all constellations, while, algorithm1outperforms algorithm- 4 by $0.7 \mathrm{~dB}$ for all constellations. Also, algorithm- 4 outperforms algorithm- 3 by $2.3 \mathrm{~dB}$ for all constellations. Furthermore, the results of circular constellations are improved over the results of rectangular one by $0.5 \mathrm{~dB}$ for all algorithms.

Also, another tests achieved on Figure 3 considering rectangular constellation only to obtain curves of SER versus S/N. Figure 4 illustrates that at SER of $10^{-5}$, the system with algorithm-2 outperforms that with algorithm- 1 by $0.6 \mathrm{~dB}$, while, the system with algorithm1 outperforms that with algorithm- 4 by $0.2 \mathrm{~dB}$. In addition, the performance of the system with algorithm- 4 is better than that with algoritm-3 by $0.6 \mathrm{~dB}$.

Table 1. Performance of ADPCM algorithms

\begin{tabular}{|c|c|c|c|c|}
\hline \multirow{2}{*}{ ADPCM } & \multicolumn{4}{|c|}{ Constellations } \\
\cline { 2 - 5 } & Rect & $(5,11)$ & $(4,12)$ & $(8,8)$ \\
\hline $\begin{array}{c}\text { Algorithm-1 } \\
\text { S/N }(\mathrm{dB})\end{array}$ & 27.5 & 28 & 27.9 & 27.9 \\
\hline $\begin{array}{c}\text { Algorithm-2 } \\
\text { S/N }(\mathrm{dB})\end{array}$ & 29.7 & 30.2 & 30.1 & 30.1 \\
\hline $\begin{array}{c}\text { Algorithm-3 } \\
\text { S/N }(\mathrm{dB})\end{array}$ & 24.5 & 25 & 24.9 & 24.8 \\
\hline $\begin{array}{c}\text { Algorithm-4 } \\
\text { S/N }\end{array}(\mathrm{dB})$ & 26.8 & 27.3 & 27.2 & 27.2 \\
\hline
\end{tabular}

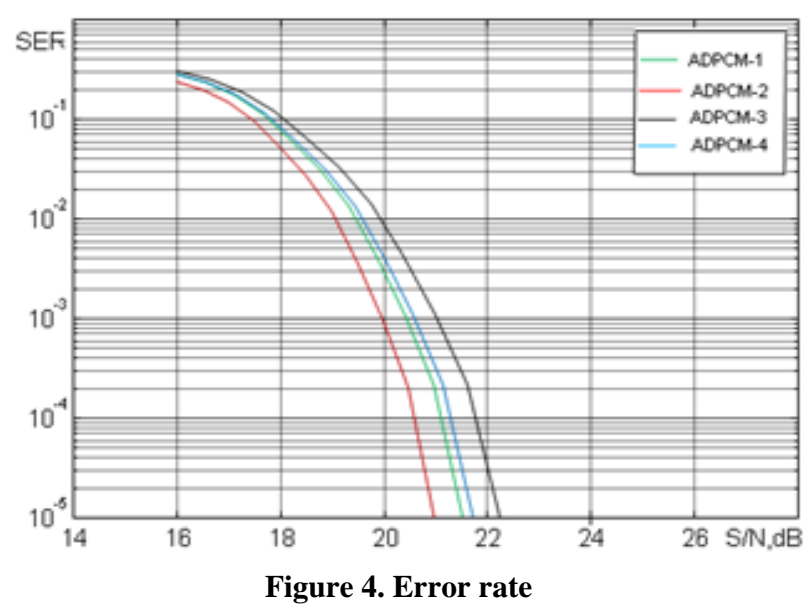

\section{CONCLUSION}

This paper developed three new algorithms of $40 \mathrm{~kb} / \mathrm{s}$ of ADPCM which were tested, and compared to the standard algorithm. The results show that algorithm-2 outperforms algorithm-1(standard algorithm), while, algorithm-1 outperforms algorithm-4, and algorithm-4 outperforms algorithm-3.

\section{REFERENCES}

[1] 40, 32, 24, $16 \mathrm{~kb} / \mathrm{s} \quad A D P C M, \quad$ CCITT Recommendation G.726-1990.
[2] Z. Markus, "Method of packet loss concealment in ADPCM Codec and ADPCM decoder with PLC circuit", U.S. Patent 20160148619, 2016.

[3] A. Gur, "Non-synchronized ADPCM with discontinuous transmission", U.S. Patent 9332585, 2016.

[4] A. Shibuta, "Wireless communication device and communication terminal", U.S. Patent 9270419, 2016.

[5] S. Ragot, "Controlling a noise-shaping feedback loop in a digital audio signal encoder avoiding instability risk of the feedback", U.S. Patent 9489961, 2016.

[6] Comparison of ADPCM algorithms, ITU-T Recommendation G.726 Appendix III-1994.

[7] M. AL-Rawi, and M. AL-Rawi, "Modified 24kb/s ADPCM System", Journal of The institution of Engineers(India): Series B(Springer), Vol.94, Issue 3, 2013.

[8] M. AL-Rawi, and M. AL-Rawi, "Comparative study of 24kb/s ADPCM algorithms", Radioelectronics and Communications Systems (Springer), Vol.57, Issue 6, 2014.

[9] M. AL-Rawi, and M. AL-Rawi, "Modified algorithm of 16kb/s ADPCM", Pacific Science Review-Elsevier, Vol.17, Issue 3, 2015.

[10] M. AL-Rawi, "Detection techniques for mitigating the nonlinear distortion of ADPCM link", Australian Journal of Multi-disciplinary Engineering (Taylor and Francis), Vol.14, Issue 1, 2018.

[11] M. AL-Rawi, and M. AL-Rawi, "Analysis and design of ADPCM system", Journal of Advanced Engineering, Vol.10, No.3, 2015.

[12] M. AL-Rawi, and M. AL-Rawi, "Performance study of multistage of $40 \mathrm{~kb} / \mathrm{s}$ ADPCM systems", International Review of Applied Sciences and Engineering (Akademiai Kiado), Vol.6, No.2, 2015. 